\title{
Jogos Sérios Ubíquos: Um mapeamento Sistemático
}

\author{
Garibaldi da Silveira Júnior - UFSM - garibaldi.dsj@gmail.com \\ Roseclea D. Medina - UFSM - roseclea.medina@gmail.com
}

\begin{abstract}
Resumo. O paradigma da computação ubíqua somada a concepção de jogos sérios possibilita que aplicações com fins instrucionais percebam e se adaptem ao contexto do jogador. Este artigo relata um mapeamento sistemático com o objetivo de identificar os trabalhos relacionados a jogos sérios ubíquos publicados nos últimos cinco anos, onde foi compreendido os principais dispositivos alvo, técnicas de avaliação dos jogos e tecnologias utilizadas no desenvolvimento deles.
\end{abstract}

Palavras-chave: jogos sérios, computação ubíqua, jogos ubíquos

\section{Ubiquitous Serious Games: A systematic review}

\begin{abstract}
The ubiquitous computing paradigm, coupled with the concept of serious games, allows instructional applications to perceive and adapt to the player's context. This article reports a systematic mapping with the objective of identifying the works related to serious games published in the last five years, which included the main target devices, game evaluation techniques and technologies used in their development.
\end{abstract}

Keywords: serious games, ubiquitous computing, ubiquitous games

\section{Introdução}

Com os avanços tecnológicos e a popularização das tecnologias digitais nas diversas áreas de atuação, os jogos digitais não estão mais restritos apenas ao lazer, Susi (2007), Ritterfeld et al. (2009) e Mitamura et al. (2012) citam como jogos sérios aqueles onde o objetivo principal está além do entretenimento, podendo estar relacionado a áreas como educação, saúde, militar etc. Eles são desenvolvidos para o aprendizado de algum tema, tal como para expandir conceitos, oferecer assistência e treinamento, que, segundo Arnab et al. (2015) fazem deles uma alternativa viável para ser usada por educadores da atual geração. Michael e Chen (2006) definem que o principal propósito dos jogos sérios é que o jogador aprenda algo enquanto está interagindo com o jogo, e se possível, se divirta no processo.

A utilização de jogos sérios na educação tende a promover grande aumento na motivação dos alunos para o aprendizado, mas, ela deve ser elencada junto a uma abordagem educacional propícia, e trabalhada em grupos, para que o efeito motivacional causado seja significante (WOUTERS et al., 2013). No entanto, segundo Witthon (2014), os jogos sérios podem não apresentar resultados satisfatórios em todas as situações, com todos os alunos ou com qualquer professor, se faz necessário que a oportunidade condiga com a ferramenta e metodologia a ser utilizada.

Um fator que faz dos jogos sérios uma alternativa para o aprendizado é a imersão, Zyda (2005) cita como imersão a sensação de presença no ambiente simulado permitido ao jogador, através de estímulos sensoriais, proporcionados através de 
gráficos, sons, interação entre os jogadores ou elementos do jogo, Faiola et al. (2013) e Hamari et al.(2016), usa o termo flow como um fator que incentiva a motivação obtida com o engajamento em um ambiente educacional baseado em jogos, facilitando o aprendizado de temas complexos.

Os autores Liu e Hwang (2010) descrevem o avanço das tecnologias na educação em rede, partindo do e-learning (electronic learning), onde as redes de computadores eram introduzidas como recurso educacional, para o m-learning(mobile learning), caracterizado pela educação a qualquer lugar e qualquer momento. Após isso, o m-learning evoluiu para o u-learning (ubiquitous learning), onde a computação procura manter-se invisível nos processos de aprendizagem, com sistemas definidos pela adaptação de conteúdo aos diversos contextos do usuário, como localização, conhecimento, habilidades, temperatura etc. Esse avanço permitiu que os jogos sérios ganhassem novos panoramas. $\mathrm{O}$ aprendizado através de jogos sérios ubíquos traz as características de imersão e engajamento propostas pelos jogos sérios, somados aos aspectos do u-learning, como a utilização de tecnologias móveis, e sistemas sensíveis a contexto, com adaptação de conteúdo referente a situação na qual o aluno se encontra. Em um ambiente de u-learning, o aprendizado está ocorrendo em todo o espaço, sem que seja percebido, os alunos têm a liberdade de aprender conforme seus próprios estilos de aprendizagem tal como garantir a flexibilidade da utilização de dispositivos móveis e de tecnologias sem fio.

Desta forma, este artigo pretendeu, através de um mapeamento sistemático da literatura, levantar dados referentes ao atual panorama de pesquisa envolvendo jogos sérios ubíquos dos últimos cinco anos. A partir desse objetivo, foram extraídas informações alusivas a captura e tratamento dos dados relativos ao contexto do usuário, a fim de buscar um estado da arte da literatura a ser utilizada como suporte a criação de um framework ${ }^{1}$ para desenvolvimento de jogos sérios ubíquos.

O termo jogos sérios ubíquos ainda não possui uma interpretação totalmente consolidada, portanto, para esta pesquisa, serão entendidos por ubíquos aqueles jogos sérios que como descritos por Klopfer (2012), permitam a mobilidade do jogador, e busquem através de alguma tecnologia ou sensor, dados relacionados ao contexto do usuário ou ambiente, e tratam estes dados de forma a facilitar a interação e adaptar o próprio conteúdo do jogo às necessidades do usuário.

$\mathrm{O}$ presente estudo está dividido da seguinte forma. Na seção dois, serão apresentadas informações referentes ao mapeamento sistemático realizado, suas etapas e resultados obtidos, na seção três é realizada a discussão referente aos dados adquiridos com a leitura dos estudos, logo após, são apresentadas as conclusões finais deste artigo.

\section{Mapeamento Sistemático}

O processo de mapeamento sistemático, definido por Kitchenham (2004), é um meio para identificar, avaliar e interpretar todos os trabalhos relevantes relacionadas a uma questão de pesquisa particular em um determinado recorte temporal, de uma forma organizada e definida por etapas, proporcionando confiabilidade ao processo e aos

\footnotetext{
${ }^{1}$ Seguindo o conceito de Braga (2002), um framework tem como principal objetivo facilitar o reúso de software, possibilitando que alternativas possam ser criadas a partir de um mesmo domínio.
} 
resultados do mapeamento.

\subsection{Planejamento}

$\mathrm{Na}$ etapa de planejamento, foram identificadas as necessidades de pesquisa e o protocolo da revisão montado. O trabalho tem como objetivo principal "identificar trabalhos relacionados a jogos sérios ubíquos", e a partir desse objetivo, foram formuladas questões de pesquisa.

Q1: Como está sendo feita a entrada de dados no sistema?

Q2: Como os dados estão sendo tratados pelo sistema?

Q3: Como estão sendo avaliados os jogos?

Q4: Quais tecnologias estão sendo utilizadas no desenvolvimento dos jogos?

Essas questões foram desenvolvidas para analisar o atual cenário de desenvolvimento de jogos ubíquos, analisando quais possibilidades e necessidades são esperadas para a área.

Para a consulta bibliográfica, foram estipulados critérios de inclusão e exclusão, que delimitam o processo de aceitação de um artigo para a próxima fase do mapeamento, tais critérios são descritos na Tabela 1 .

\section{Tabela 1. Critérios de inclusão e exclusão.}

\begin{tabular}{|l|l|}
\hline \multicolumn{1}{|c|}{ Inclusão } & \multicolumn{1}{|c|}{ Exclusão } \\
\hline $\begin{array}{l}\text { (I1) Relatos de desenvolvimento de jogos } \\
\text { sérios ubíquos; }\end{array}$ & $\begin{array}{l}\text { (E1) Trabalhos não relacionados a jogos } \\
\text { sérios; }\end{array}$ \\
\hline $\begin{array}{l}\text { (I2) Frameworks ou métodos de de } \\
\text { desenvolvimento de jogos sérios ubíquos; }\end{array}$ & $\begin{array}{l}\text { (E2) Trabalhos relacionados a jogos sérios } \\
\text { sem conceitos de computação ubíqua; }\end{array}$ \\
\hline $\begin{array}{l}\text { (I3) Trabalhos Relacionados a avaliação } \\
\text { de jogos sérios ubíquos; }\end{array}$ & $\begin{array}{l}\text { (E3) Trabalhos com data de publicação } \\
\text { anterior ao ano de 2012; }\end{array}$ \\
\hline $\begin{array}{l}\text { (I4) Pesquisas relacionadas a jogos sérios } \\
\text { ubíquos. }\end{array}$ & $\begin{array}{l}\text { (E4) Trabalhos incompletos ou sem versão } \\
\text { completa disponível; }\end{array}$ \\
\hline & $\begin{array}{l}\text { (E5) Trabalhos que não estejam } \\
\text { publicados na língua inglesa ou } \\
\text { portuguesa. }\end{array}$ \\
\hline & (E6) Estudos duplicados \\
\hline
\end{tabular}

A partir disso, foi estipulada a string de busca, usando os operadores lógicos OR e AND, permitindo uma abrangência de sinônimos dos termos que possuem maior relevância neste estudo. A string utilizada pode ser observada abaixo.

"game" AND ("based learning" OR "educational" OR "learning" $O R$ "teaching” OR "training” OR "serious" OR "instructional”) AND ("ubiquitous" OR "adaptive" OR "pervasive" OR "context aware" OR "context sensitive") 
Para a procura de artigos em português, foi utilizada a seguinte string.

"jogo" AND ("aprendizado baseado em jogos" OR "educacional” $O R$ "aprendizado" OR "ensino" OR "treinamento" OR "serio" OR "instrucional”) AND ("ubiquo” OR “adaptativo” OR "pervasivo” OR "sensivel a contexto")

Para a pesquisa bibliográfica, foram selecionadas as bibliotecas digitais IEEE Xplore, ACM Digital e o Banco de Teses \& Dissertações da Capes, onde foi aplicado a string de busca. A string foi adaptada, sem modificar sua estrutura, conforme o motor de busca, para que sejam buscados apenas estudos com os termos incluídos no título, resumo e palavras-chave dos artigos.

Além disso, foram incluídos artigos por busca manual dos eventos SBIE, Ubicomp e do periódico RENOTE. Foram delimitados apenas estudos com ano de publicação equivalente ou superior ao de 2012. Dentre os resultados da busca inicial, foram selecionados o total de 598 estudos.

\subsection{Condução}

Nessa etapa, foi realizado a leitura do título, palavras-chave e resumo de cada um dos artigos selecionados até o momento. Os artigos foram analisados seguindo os critérios de inclusão e exclusão, dessa forma reduzindo o montante de estudos para 183, excluindo da lista os que se encaixam em pelo menos um critério de exclusão.

Foi realizada a leitura da introdução e conclusão dos artigos selecionados após a análise dos critérios de inclusão e exclusão, permitindo um refinamento mais apurado quanto aos estudos até então selecionados para este trabalho, dada essa etapa, o montante de estudos foi reduzido para 90, onde estes foram lidos na íntegra, e analisados a partir das questões norteadoras deste trabalho, promovendo uma análise do panorama atual de pesquisas relacionadas a jogos sérios ubíquos.

Q1: Como está sendo feita a entrada de dados no sistema?

A computação ubíqua tem por sua principal característica a mobilidade e o intuito de esconder os processos educacionais da percepção do usuário. Para tanto, são utilizados tecnologias que possibilitem a captura dos dados referentes ao contexto em que o usuário se encontra, estes dados podem ter relação a sua localização física, aspectos físicos do ambiente onde o mesmo se encontra, percepções referentes às características de aprendizado do usuário etc, realizando uma troca de dados constante entre os dispositivos e o ambiente, com o mínimo de interação possível do usuário (PIMENTA, 2014). Toda essa demanda pode ser obtida através de sensores, que monitoram as atividades realizadas pelo usuário durante o jogo, no entanto, devido a numerosa quantidade de tipos diferentes de dados, a variedade de sensores utilizados também é alta. Com isso, se fez necessário analisar como está sendo feita a captura destes dados nos jogos sérios ubíquos desenvolvidos, permitindo o conhecimento sobre os principais dispositivos e sensores. 


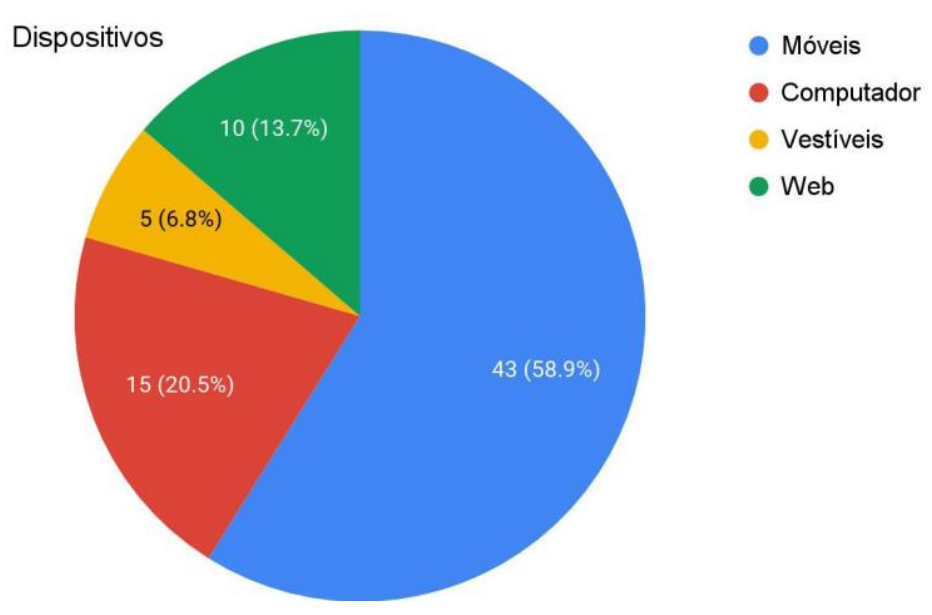

Figura 1. Dispositivos

Dentre os artigos selecionados, 73 deles possuíam relação direta com algum tipo de dispositivo, os outros 17 representavam um estudo bibliográfico, metodologia ou framework que abrangia o conceito sem focar em alguma tecnologia específica. $\mathrm{Na}$ figura 1, pode ser percebido que os dispositivos móveis representam 43 dos trabalhos que relatam o desenvolvimento ou aplicação de um jogo sério ubíquo, nessa categoria, foram considerados dispositivos como smartphones, tablets e notebooks. Seguindo, os computadores do estilo desktop representam 15 dos estudos, plataformas web são encontradas em 10 estudos e os dispositivos vestíveis fazem parte de cinco dos estudos analisados.

Além do dispositivo principal, que suporta o jogo e permite a sua utilização, tem-se os sensores, que captam as informações e trazem a modularidade de um jogo sério ubíquo, não se restringindo a uma única possibilidade.

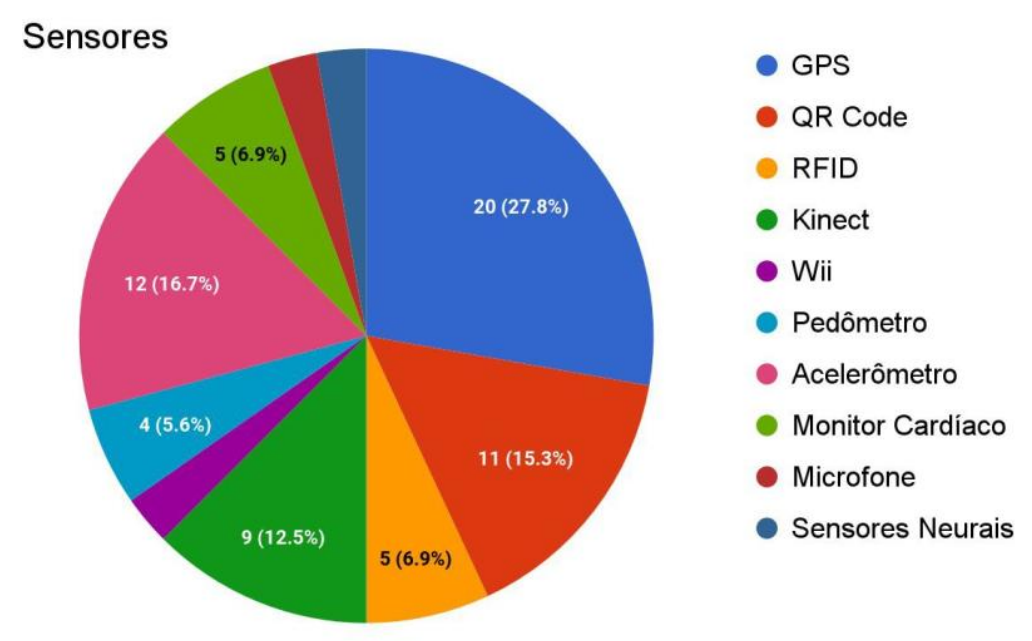

Figura 2. Sensores

Dentre os sensores usados nos estudos selecionados, podem ser visualizados na Figura 2, aqueles que buscam dados referentes a localização física do usuário: O GPS representou 20 dos artigos selecionados, também foram encontrados jogos que utilizam RFID em cinco estudos e QRCode em 11. Dos sensores que estão relacionados ao 
monitoramento gestual e de atividades: O Kinect representa 9 estudos, partindo da mesma premissa que o Wii, que representa dois, também foram analisados 12 artigos que usam o Acelerômetro, 4 o Pedômetro, 5 o Monitor Cardíaco e 2 Sensores Neurais. Dois dos estudos usaram o microfone para reconhecimento de voz e tonalidade vocal.

Q2: Como os dados estão sendo tratados pelo sistema?

O tratamento dos dados permite que o aspecto de inteligência e ubiquidade de um jogo sério possa ser alcançado. Durante a análise dos artigos, foi percebido que alguns dos modelos de tratamento de dados seguiam um mesmo padrão, sendo eles:

- Feedback de performance: O sistema de feedback transmite ao jogador a sensação de recompensa, e permite o engajamento e a motivação para continuar jogando. Dentro dos jogos sérios ubíquos, esse sistema vem com o acompanhamento da performance, medindo a evolução do jogador através dos dados obtidos pelos sensores, podendo ser a quantidade de passos dados durante uma atividade, realização de um gesto corretamente, conclusão de uma atividade proposta etc. A recompensa normalmente é estabelecida em pontuação, algum item ou moeda virtual, que pode ser utilizado dentro do jogo etc, estimulando a competitividade entre os jogadores e trazendo a sensação de objetivo a atividade realizada.

- Análise de atividade: Nessa categoria, se encaixam os jogos ubíquos que procuraram uma adaptação automática do seu conteúdo conforme a utilização do usuário, buscando as fragilidades e necessidades do mesmo quanto a utilização, seja diminuindo a dificuldade para que o jogador não se sinta frustrado ao jogar, ou aumentando a mesma, permitindo um desafio maior.

- Questionário: Os questionários abordam uma adaptação de conteúdo pré estabelecida, seja no formato de perguntas que demonstram o propósito adaptativo ou através de diálogos da narrativa do jogo, que permitem que o processo seja camuflado. A partir de determinado ponto, pode ser descoberto o perfil do usuário, é definido como deve ser feito o andamento do jogo, para que o mesmo consiga interagir da melhor forma.

Q3: Como estão sendo avaliados os jogos?

Da seleção dos artigos aceitos, 54 deles envolviam a avaliação dos jogos sérios propostos de alguma maneira, alguns deles, por tratar-se de um estudo preliminar, abordavam ou somente a estrutura conceitual, ou relataram a etapa de desenvolvimento da proposta. Foi percebido que 28 dos artigos realizaram a avaliação para medir o nível de motivação, engajamento e satisfação gerado após a aplicação prática do jogo sério, em um circuito pré determinado ou dando liberdade ao jogador. Além disso, 31 artigos também relataram a utilização de métodos avaliativos que verificaram o nível de aprendizado adquirido com a utilização dos jogos sérios desenvolvidos em comparação a uma abordagem de ensino tradicional, essa avaliação é realizada dividindo por grupos de alunos que não possuem conhecimento anterior, que jogaram após obter o 
conhecimento e aqueles que aprenderam da maneira tradicional após jogar, com isso verificando as variáveis possíveis. Quanto ao método de aplicação, 35 estudos usam o formato de questionário durante o processo de aplicação do jogo sério com um grupo de alunos, os outros 19 realizaram a avaliação através da observação de pesquisa de campo.

\section{Q4: Quais tecnologias estão sendo utilizadas no desenvolvimento dos jogos?}

O desenvolvimento de jogos sérios ubíquos não se limita a utilização de uma abordagem única, e tão pouco ferramentas. Para tanto, são criados frameworks, que facilitam o trabalho do desenvolvedor, seja entregando ao mesmo um arcabouço teórico do projeto a ser criado, ou também uma série de códigos e elementos modulares que podem ser reutilizados conforme a necessidade do jogo. Essa questão de pesquisa visou descobrir quais ferramentas e frameworks foram desenvolvidos e estão sendo utilizadas para a criação de tais jogos, suas potencialidades e limitações.

O trabalho de Lumpoon \& Thiengburanathum (2016) trata de um framework de desenvolvimento de jogos sérios sensíveis ao contexto de localização. No estudo, é criado um jogo para dispositivos móveis com o objetivo de promover o engajamento de turistas no conhecimento da cultura local da cidade de Chengdu, onde são separados grupos, que tem um tempo determinado para conhecer sobre os pontos turísticos usando o sistema de GPS do smartphone, e responder questões sobre eles, acumulando pontos com isso, e no final do tempo estipulado, gerando um placar. O sistema foi desenvolvido através da plataforma híbrida Ionic, usando uma arquitetura cliente-servidor.

O estudo de Chen \& Shih (2012) relata a criação de um metamodelo para o desenvolvimento de jogos instrucionais pervasivos, baseado em três princípios: pedagógico, contexto e design, onde, a partir deles, foram estipulados questões para servirem como guia e suporte teórico no desenvolvimento desses tipos de jogos.

Com relação a jogos adaptativos em dispositivos móveis, o framework desenvolvido por Szegletes \& Forstner (2013) busca um sistema de recompensas inteligente, baseado na expectativa do usuário pela mesma. A estrutura criada utiliza de sensores neurais para capturar os dados do jogador, como a concentração dele, expressões faciais e sinais biológicos, traçando o seu perfil e oferecendo uma recompensa que não prejudique o estímulo proporcionado pelo jogo.

São utilizadas plataformas de desenvolvimento para dispositivos móveis na maioria dos artigos selecionados, 23 deles usavam ferramentas como Android Studio, Ionic e Phonegap, foi percebido um recorrente aumento do uso daquelas consideradas híbridas $^{2}$ nos últimos anos quando comparado às nativas. Além disso as engines de jogos são usadas em 15 dos estudos, entre elas: Unity, Unreal Engine, GameMaker Studio, RPG Maker, e também os mundos virtuais OpenSimulator e Second Life.

\footnotetext{
${ }^{2}$ Ferramentas para desenvolvimento híbrido mesclam as tecnologias presentes nas aplicações para web e com o código nativo, permitindo que a aplicação seja compatível com vários dispositivos e utilize todos os recursos e sensores disponíveis.
} 


\section{Discussão}

Esta seção discute os resultados obtidos a partir do processo de mapeamento sistemático, apontando o conhecimento produzido com ênfase nas questões que nortearam esta pesquisa.

Quanto aos dispositivos e sensores utilizados para a entrada de dados, foi percebido que aqueles considerados móveis estão em mais de 50\% dos artigos, com isso, pode ser considerado a importância da mobilidade nos jogos sérios ubíquos, e também o principal foco dos mesmos. Além disso, os jogos para dispositivos vestíveis representaram a menor parcela dos estudados, no entanto, os mesmos mostraram-se recentes, indicando um fator de crescimento e possibilidade de futuras pesquisas usando eles.

Os sensores mostraram-se diversificados e abrangendo aplicações para alguns propósitos, como a educação, condicionamento físico e mental e reabilitação motora, onde aqueles que buscavam o contexto de localização se mostraram em maior número, podendo ser constatado que esta informação está diretamente ligada a popularidade dos dispositivos móveis e os sensores embutidos na estrutura deles. Além disso, a presença de pesquisas que relatem a utilização de sensores biológicos mostra uma premissa do potencial e das possibilidades que um jogo sério ubíquo pode alcançar.

A partir das informações levantadas referente ao tratamento dos dados, foi possível entender e classificar como o jogo se comporta para adaptar-se a necessidade do jogador. Dentre os métodos vistos, aqueles que se aplicam em formato de questionário mostraram-se em maior número e de forma mais simples, no entanto, são limitados, visto que abrangem apenas as possibilidades de adaptação preestabelecidas. Já aqueles que possuem a adaptação automática pela atividade do usuário, através da análise e apuração das informações obtidas por um banco de dados, tendem a absorver melhor o perfil do jogador, no entanto, sua aplicação e o processo de desenvolvimento é mais complexo, envolvendo muitas vezes sensores que descaracterizam a portabilidade e mobilidade do jogo.

Quanto aos métodos de avaliação, foi percebido que os que utilizam questionários podem produzir boas respostas qualitativas quanto ao comportamento do jogo e possibilitar que melhorias possam ser efetuadas, no entanto, aqueles que são baseados em uma pesquisa quantitativa podem não ser tão efetivos para a precisão dos resultados, para uma análise desse tipo, a utilização de métodos automáticos de avaliação ou a presença de uma avaliação por observação de comportamento pode demonstrar melhores resultados.

$\mathrm{O}$ conhecimento quanto às tecnologias que estão sendo utilizadas no desenvolvimento de jogos ubíquos possibilitou visualizar as suas características necessárias, segundo Klopfer (2012), eles devem exigir o mínimo de recursos computacionais possíveis para poder funcionar na maioria dos dispositivos utilizados hoje em dia, como smartphones, tablets e computadores. Com isso, em sua maioria, os mesmos não utilizam motores gráficos $3 \mathrm{D}$, que irão exigir maior processamento de vídeo e armazenamento, também gerando um consumo maior de bateria dos dispositivos móveis. Nesses casos, é optado pela utilização de ferramentas que possibilitam o desenvolvimento de jogos $2 \mathrm{D}$, diminuindo o consumo dos recursos. 
Além disso, as ferramentas devem possuir integração aos sensores, ou possibilidade de extensão através de código aberto.

\section{Conclusão}

A utilização de jogos sérios na realização e simulação de atividades que envolvem uma prática onde é necessário a imersão do usuário no ambiente, representa uma grande fonte de pesquisas em áreas relacionadas à educação e o treinamento. Jogos sérios podem apresentar um conteúdo de difícil representação no mundo real, de uma maneira que venha a prender a atenção do usuário, trazendo interatividade ao processo de aprendizado. Quando adicionado a característica de ubiquidade a eles, a imersão ao ambiente é potencializada, não limitando sua utilização a um espaço físico e adicionando transparência a computação envolvida no processo, transformando a experiência e adaptando conforme a necessidade e situação do jogador.

Este artigo relatou o processo de um mapeamento sistemático da literatura, com o objetivo de identificar estudos relacionados a jogos sérios ubíquos. A partir desse estudo foi possível relacionar e compreender os processos metodológicos, ferramentas e tecnologias usadas no desenvolvimento e avaliação de jogos sérios ubíquos. O maior enfoque mantém-se nos dispositivos móveis, mas o número de pesquisas relacionadas a jogos sérios para dispositivos vestíveis tem aumentado.

De acordo com as informações obtidas, ainda existem possibilidades a serem alcançadas nesse campo, alguns estudos remetem como trabalhos futuros uma análise mais apurada quanto ao benefício educacional das ferramentas, outros procuram aumentar a portabilidade da aplicação, atingindo maior número de dispositivos, outros buscam explorar a realidade aumentada e a realidade virtual nos jogos desenvolvidos. Como trabalhos futuros, é pretendido a criação de um framework para o desenvolvimento de jogos sérios ubíquos, categorizando os principais elementos que os compõem além de formas e métodos para chegar em um resultado com as características que fazem um jogo sério ser considerado ubíquo.

\section{Referências}

ARNAB, S., LIM, T., CARVALHO, M. B., BELlOTTI, F., FREITAS, S., LOUCHART, S., ... \& DE GLORIA, A. (2015). Mapping learning and game mechanics for serious games analysis. British Journal of Educational Technology, 46(2), 391-411.

BRAGA, R. T. V. (2002). Um processo para construção e instanciação de frameworks baseados em uma linguagem de padrões para um domínio específico (Tese de doutorado , Universidade de São Paulo).

CHEN, C.P. AND SHIH, J.L. (2012) A prototype on a meta-model for designing instructional pervasive games. In Digital Game and Intelligent Toy Enhanced Learning (DIGITEL), 2012 IEEE Fourth International Conference on (pp. 47-51). IEEE.

CSIKSZENTMIHALYI, M. (1997). Finding flow: The psychology of engagement with everyday life. New York: HarperCollins. 
FAIOLA, A., NEWLON, C., PFAFF, M., \& SMYSLOVA, O. (2013). Correlating the effects of flow and telepresence in virtual worlds: Enhancing our understanding of user behavior in game-based learning. Computers in Human Behavior, 29(3), 1113-1121.

HAMARI, J., SHERNOFF, D. J., ROWE, E., COLLER, B., ASBELL-CLARKE, J., \& EDWARDS, T. (2016). Challenging games help students learn: An empirical study on engagement, flow and immersion in game-based learning. Computers in Human Behavior, 54, 170-179.

KITCHENHAM, B. (2004). Procedures for performing systematic reviews. Keele, UK, Keele University, 33(2004), 1-26.

KLOPFER, E.; SHELDON, J.; PERRY, J.; CHEN, V. H. (2012) Ubiquitous games for learning (UbiqGames). Journal of Computer Assisted Learning, v. 28, 465-476.

LIU, G. Z., \& HWANG, G. J. (2010). A key step to understanding paradigm shifts in e-learning: towards context-aware ubiquitous learning. British Journal of Educational Technology, 41(2), E1-E9.

LUMPOON PN, THIENGBURANATHUM P. (2016) Effects of integrating a mobile game-based learning framework in a cultural tourism setting. InSoftware, Knowledge, Information Management \& Applications (SKIMA), 10th International Conference on 2016 Dec 15 (pp. 281-285). IEEE.

MICHAEL, D. \& CHEN, S. (2006) Serious games: Games that educate, train, and inform. Boston, MA.: Thomson Course Technology.

MITAMURA, T.; SUZUKI, Y.; OOHORI, T. (2012). Serious games for learning programming languages. In: Systems, Man, and Cybernetics (SMC), 2012 IEEE International Conference on, pp.1812, 1817, 14-17.

PIMENTA, MATHEUS CSC, ET AL. A game engine for building ubigames. Proceedings of the 13th Annual Workshop on Network and Systems Support for Games. IEEE Press, 2014.

RITTERFELD, U., CODY, M., \& VORDERER, P.. (2009). Serious games: Mechanisms and effects. Routledge.

SUSI, T., JOHANNESSON, M., \& BACKLUND, P. (2007). Serious games: An overview.

SZEGLETES, L., \& FORSTNER, B. (2013). Reusable framework for the development of adaptive games. In Cognitive Infocommunications (CogInfoCom), 2013 IEEE 4th International Conference on (pp. 601-606). IEEE.

WHITTON, N. (2014). Digital games and learning: Research and theory. Routledge. WOUTERS, P., VAN NIMWEGEN, C., VAN OOSTENDORP, H., \& VAN DER SPEK, E. D. (2013). A meta-analysis of the cognitive and motivational effects of serious games.

ZYDA, M. (2005). From visual simulation to virtual reality to games. Computer,38(9), 25-32. 